\title{
Eye-movement patterns do not mediate size distortion effects in hemispatial neglect: looking without seeing
}

\author{
Monika Harvey ${ }^{\mathrm{a}, *}$, Iain D. Gilchrist ${ }^{\mathrm{b}}$, Bettina Olk ${ }^{\mathrm{c}}$, Keith Muir ${ }^{\mathrm{d}}$ \\ a Department of Psychology, University of Glasgow, Glasgow G12 8QB, Scotland, UK \\ ${ }^{\mathrm{b}}$ Department of Experimental Psychology, University of Bristol, Bristol, England, UK \\ ${ }^{\mathrm{c}}$ Department of Psychology, University of British Columbia, Vancouver, BC, Canada \\ d Department of Neurology, Southern General Hospital, Glasgow, Scotland, UK
}

Received 3 July 2002; received in revised form 31 October 2002; accepted 4 November 2002

\begin{abstract}
Over the last decade a range of studies have shown that some patients with hemispatial neglect subjectively underestimate the size of objects presented in their contralesional hemispace. Recently, it has been suggested that the effect is simply due to either hemianopia [Brain 124 (2001) 527], or the combination of neglect and hemianopia [Neurology 52 (1999) 1845]. In the current study we asked right hemisphere lesioned patients with and without neglect and hemianopia as well as healthy controls to judge either two horizontal or vertical lines presented simultaneously in right and left hemispace and monitored their eye movements. Three out of the six patients showed the predicted size distortion effect for horizontal lines. We found no evidence that the effect was mediated by eye movements. The two neglect patients who showed the strongest left side underestimation showed symmetrical (left, right) scanning of the lines both in terms of number of fixations and fixation time, yet they still failed to judge the relative size veridically. In addition, we did not find strong evidence for a link with hemianopia. We therefore propose that the effect reflects a computational/representational failure of processing for horizontal extent. (C) 2002 Elsevier Science Ltd. All rights reserved.
\end{abstract}

Keywords: Hemispatial neglect; Size distortion; Eye movements

\section{Introduction}

Hemispatial neglect is a debilitating condition which can occur following damage to a variety of brain areas, but has been chiefly associated with damage involving the parieto-temporo-occipital junction of the right cortical hemisphere [29] although it has recently been argued that it is mainly the right temporal lobe that mediates the disorder [21]. Patients displaying the syndrome generally fail to respond appropriately towards stimuli located in the contralesional space and may ignore stimuli or reduce the extent of eye and hand movements to objects or events occurring within this space.

In addition to these symptoms, over the last decade, several authors have also reported evidence for a distortion of visual space in such patients $[3,12,13,19,26,28]$, a phenomenon initially described by Gainotti and Tiacci in 1971 [9]. The patients perceive two identical horizontally arranged objects as different in size with the left object ap-

\footnotetext{
* Corresponding author. Tel.: +44-141-330-6174; fax: +44-141-330-4606

E-mail address: m.harvey@psy.gla.ac.uk (M. Harvey).
}

pearing subjectively shorter than the right one. Moreover, such patients fail to place end-points of an imagined or partially presented line correctly, misplacing the end-points so that a longer segment lies on the contralesional side of the midpoint and a shorter segment on the ipsilesional side.

Bisiach argues that the misperception may be a consequence of progressive contralateral relaxation of the medium for space representation [4]. In recent papers, Milner et al. [25,27] and Irving Bell et al. [19] have argued for a similar computational failure as the underlying cause of this size distortion. Further support for this argument comes from a recent study by Harvey et al. [14] who found that neglect patients underestimated the length of even a single line presented in left space.

In contrast to these explanations, both Ferber and Karnath [7] and Doricchi and Angelelli [5] have argued that the distortion effect is simply mediated by hemianopia. Ferber and Karnath [7], reporting some size distortion effects in patients with pure neglect and no hemianopia, found strongest impairments in patients with left hemianopia but no neglect. Doricchi and Angelelli [5] used the endpoint task and found that only patients with both neglect and hemianopia 
overextended the distance contralesionally and underextended it ipsilesionally.

Studies that have monitored eye-movement patterns in patients with neglect and hemianopia have indeed found hypometric scanning towards the blind hemifield. Barton et al. [2] monitored eye movements in a line bisection task and found that hemianopic patients showed a fixation peak at the contralateral end of the line as well as the centre of the line, whereas for the neglect patients, who again all had visual field defects, all measures of scanning position in space were displaced rightwards. The presence of a hemianopia might cause size misinterpretation because part of the stimulus falls in the hemianopic field, and is not detected and would not be the subject of an inspection saccade. Such patients may simply not scan the left one of two simultaneously presented lines or scan it only partly and therefore judge the left line as relatively shorter than the right one.

Somewhat counterintuitively Doricchi and Angelelli [5] also claim that scanning patterns may be responsible for the observed size impairment. However, they speculate that when confronted with the endpoint task, such patients shift the centre of their gaze and attention toward the ipsilesional position where the dot has to be placed, failing to compensate for the progressive absence of retinotopical information from the blind hemifield. According to the authors, this induces a hypometric exploration of the ipsilesional side leading to ipsilesional underextension in distance reproduction. The opposing effect accounts for the contralateral overextension: in this case lack of retinal input from the left hemifield triggers hypermetric compensatory scanning towards the blind hemifield which the damaged right hemisphere may be unable to correct for, thus leading to overextension. This explanation was of course speculative as eye movements were not monitored in the study.

The current study was designed to address whether eye-movement behaviour does correlate with the described size distortion effect and to investigate the nature of this relationship. If the effect is a more fundamental object processing failure as argued later by Milner [25] and also by Irving-Bell et al. [19] then it should be independent of eye-movement patterns.

We therefore monitored eye movements in a task in which either two horizontal or vertical lines of either identical or non-identical length were presented to the subject one in the left, one in the right hemispace. We chose this task as, unlike the endpoint task, it controls for the effect of directional hypokinesia $[16,17]$ avoiding the influence of such potential motor impairments on the results. Right hemisphere lesioned neglect patients with and without hemianopia as well as right hemisphere lesioned patients without neglect and healthy control subjects were tested.

\section{Method}

\subsection{Subjects}

Four patients with unilateral right hemisphere infarct who all performed outside normal limits on the formal subtests of the Behavioural Inattention Test (BIT, [30]) and showed clear evidence of neglect of the left hemispace and two patients with unilateral right hemisphere infarct (RCVA) who showed no sign of hemispatial neglect were tested (see Fig. 1 for lesion mapping of all patients). Demographic and clinical information is given in Table 1. Hemianopia was assessed with clinical confrontation testing, the procedure was the same for all patients. Six healthy elderly participants matched in age and education to the neglect patients (mean age $=73.8$, S.D. $=4.3$ ) with no reported history of neurological or cardiovascular problems, were also tested. All participants were right-handed as assessed by the administration of the Annett Handedness Inventory [1] and had normal or corrected to normal vision in both eyes.

\subsection{Displays and procedure}

Displays were generated and presented on an IBM compatible personal computer with a $17 \mathrm{in}$. VGA monitor (133 MHz Compaq Deskpro). The programs controlling the displays were written in-house. The display consisted of either two horizontal or two vertical lines arranged on either side of the fixation point starting either $1.92^{\circ}$ from the fixation point in terms of the horizontal lines or $11.4^{\circ}$ from this point in the case of the vertical lines. The fixation point was a white circle on black background with a diameter of $0.5^{\circ}$. All lines were solidly drawn and appeared white on a black background. They were either identical in length in which case the horizontal lines subtended $7.7^{\circ}$ of visual angle horizontally and $0.23^{\circ}$ vertically. The identical vertical lines subtended $7.7^{\circ}$ of visual angle vertically and

Table 1

Clinical details and test performances of the six patients

\begin{tabular}{|c|c|c|c|c|c|c|}
\hline Patient & Age & Lesion location & Poststroke (month) & Hemianopia & Extinction & BIT \\
\hline $\mathrm{LC}$ & 79 & $\mathrm{R}$ occipital, inferior temporal and basal ganglia & 42 & Yes & No & 115 \\
\hline JR & 73 & $\mathrm{R}$ temporo-parietal & 20 & No & No & 112 \\
\hline MW & 63 & $\mathrm{R}$ fronto-temporo-parietal & 9 & Yes & Yes & 119 \\
\hline RA & 54 & $\mathrm{R}$ temporo-parietal, basal ganglia & 8 & No & No & 122 \\
\hline MH & 75 & $1 \mathrm{R}$ frontal, $2 \mathrm{R}$ parietal & 13 & No & No & 140 \\
\hline $\mathrm{AQ}$ & 66 & $\mathrm{R}$ fronto-parietal & 15 & No & No & 144 \\
\hline
\end{tabular}

Scores below 129 on the BIT indicate hemispatial neglect. 
LC

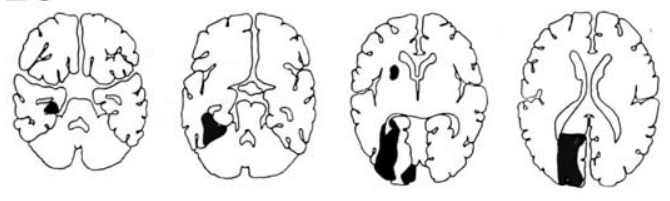

\section{JR}

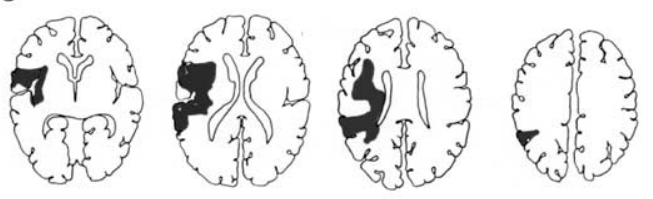

MW

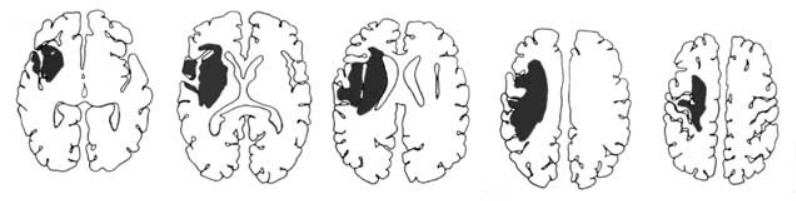

\section{RA}

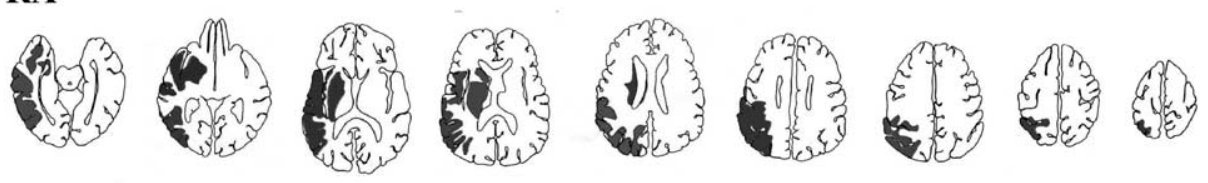

\section{MH}
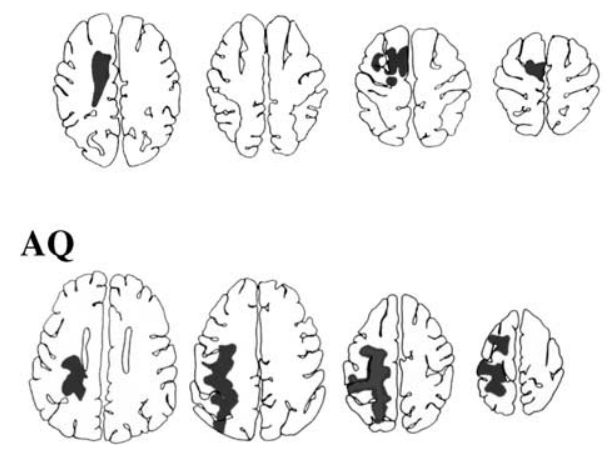

Fig. 1. Individual CT maps of damaged areas of the six patients. The figure shows: for AQ slices $8-11$ and for RA slices $3-11$ (at $0^{\circ}$ ), for MH slices 10,12 , and $13\left(\right.$ at $5^{\circ}$ ), for MW slices 5-9 (at $10^{\circ}$ ), for JR slices 5-8 and LC slices 2, 3, 5 and 6 (at $20^{\circ}$ ). Templates were taken from Damasio and Damasio, 1989.

$0.23^{\circ}$ horizontally. These identical lines were randomly interspersed with non-identical lines: for the horizontal lines four lines each were $75 \%$ of the size of the identical line with either the right or left segment missing on either the right or left line, a further four each were $87.5 \%$ of the size of the identical line again with either the right or left segment missing on either line. The magnitude and number of the non-identical vertical lines was the same but the reduction was done at either end to make the subjects perform a length comparison rather than purely judge the difference in stimuli by height (which they would have been able to do had only the top or bottom segment been shortened). There were 62 trials ( 30 trials on lines of identical length) in each block and the distance between eyes and screen was $57 \mathrm{~cm}$.
Participants viewed the displays with both eyes, but only data of the eye with the best spatial accuracy was analysed. This was assessed using the best average spatial accuracy for each eye in the validation procedure as outlined below.

The fixation point was presented until fixation was stable. This was assessed by the experimenter who viewed a real time display of eye position superimposed on the fixation location on another computer that was linked to the eye tracker. The fixation point disappeared with stimulus presentation. Stimuli remained visible until participants pressed one of two response buttons positioned next to each other in front of them. Participants, whose heads rested in a chin rest, were falsely informed that none of the pairs of lines were identical and were asked to indicate on each trial which one 
of the two lines appeared to be longer and press the corresponding response button (either left or right). To control for response bias, all subjects were also tested under the opposing response instruction. So on half the blocks they were asked to judge which stimulus appeared longer and on the other half which appeared shorter. To prevent interference breaks were given between each block (horizontal 'long', vertical 'long', horizontal 'short', vertical 'short').

Longer breaks between conditions were needed during the testing of the patients. Also, in addition, each neglect patient completed each experimental condition twice so that there were eight blocks overall. The only exception to this was patient LC who, due to fatigue, completed only three blocks for horizontal and two blocks for vertical lines. Order of blocks was counterbalanced between subjects as completely as possible.

\subsection{Eye movement recording}

Eye movements were recorded with the SMI EyeLink System (SensoMotoric Instruments $\mathrm{GmbH}$, Teltow, Germany) using the centre of the pupil as well as the corneal reflection technique to define pupil position.

Each block of trials was preceded by a nine-point grid calibration and validation for which participants were instructed to saccade to a white circle $\left(0.5^{\circ}\right)$ on a black background, which appeared sequentially at nine points in a square array. Between trials, the fixation circle reappeared to correct for drift due to head movements. Eye movements were recorded at $250 \mathrm{~Hz}$ sample rate at a spatial resolution, typically, of $0.3^{\circ}$. Saccade onset was defined as a change in eye position with a minimum velocity of $30 \%$ s or a minimum acceleration of $8000^{\circ} / \mathrm{s}^{2}$.

Subjects had a maximum of $20 \mathrm{~s}$ to respond by button press, trials in which no button press occurred in that time were deleted. Only the neglect patients RA and MW showed this and 1.3 and $7.9 \%$ of trials were deleted, respectively.
Recording errors occurred for healthy subject S6 only, in this case no saccades were recorded in $13.3 \%$ of trials.

\section{Analyses and results}

The data was analysed separately for each subject and only the performance of the identical horizontal and vertical lines was studied in depth (for brevity only the results of the horizontal lines are presented in Tables 2 and 3). There were four dependent measures which were taken for each subject, separately for horizontal and vertical lines (albeit combined across the 'longer/shorter' instructions).

\subsection{Response bias}

Response bias (as a possible indicator for hypokinesia) was calculated as the percentage of left versus right button presses and significant differences between these two measures were assessed with the sign test. For consistency with the measurement of size distortion, response bias is presented as a contrast score ((left button presses - right button presses)/(left button presses + right button presses)), see Table 2.

Neither the patients nor the healthy controls gave any evidence of a consistent response bias in terms of directional hypokinesia which would have been reflected in contrast scores around -1 . Some participants did, however, show systematic response biases that were restricted to specific visual conditions. Neglect patient LC showed a significant rightward bias for the vertical lines but a significant leftward bias for the horizontal lines, a pattern also shown by the healthy control S2. RCVA control patient MH showed a significant leftward bias with the vertical lines as did the healthy subject S1. Like LC and S2, patients MW and $\mathrm{MH}$ and the healthy subject S6 showed significantly more leftward button presses for the horizontal lines.

Table 2

Bias scores for all patients and healthy controls for identical and non-identical horizontal lines

\begin{tabular}{lccrr}
\hline $\begin{array}{l}\text { Patient and } \\
\text { healthy control }\end{array}$ & $\begin{array}{l}\text { Response bias, } \\
\text { identical line }\end{array}$ & $\begin{array}{l}\text { Distortion bias, } \\
\text { identical line }\end{array}$ & $\begin{array}{l}\text { Distortion bias, } \\
12.5 \% \text { right, left }\end{array}$ & $\begin{array}{l}\text { Distortion bias, } \\
25 \% \text { right, left }\end{array}$ \\
\hline LC & $\mathbf{0 . 4 0}$ & $\mathbf{0 . 8 8}$ & $\mathbf{0 . 7 1}$ & $\mathbf{0 . 4 6}$ \\
JR & 0.03 & $\mathbf{0 . 6 6}$ & 0.13 & -0.13 \\
MW & $\mathbf{0 . 2 5}$ & $-\mathbf{0 . 3 8}$ & 0.23 & -0.13 \\
RA & -0.15 & $\mathbf{- 0 . 5 1}$ & $\mathbf{0 . 4 1}$ & 0.00 \\
MH & $\mathbf{0 . 2 2}$ & $\mathbf{0 . 4 8}$ & -0.19 & -0.28 \\
AQ & 0.00 & $-\mathbf{0 . 7 5}$ & 0.18 & 0.00 \\
S1 & 0.20 & -0.10 & 0.18 & -0.03 \\
S2 & $\mathbf{0 . 5 3}$ & $-\mathbf{0 . 7 7}$ & 0.00 & 0.00 \\
S3 & 0.17 & $-\mathbf{0 . 8 3}$ & 0.18 & 0.00 \\
S4 & 0.03 & -0.17 & 0.37 & -0.03 \\
S5 & -0.17 & 0.00 & -0.13 & 0.00 \\
S6 & $\mathbf{0 . 4 0}$ & &
\end{tabular}

For the non-identical lines the line was either 12.5 or $25 \%$ shorter on the right, or 12.5 or $25 \%$ shorter on the left. For the response bias, a score of 0 indicates no bias, a score of $1,100 \%$ left button presses and a score of $-1,100 \%$ right button presses. For the distortion biases a score of 0 represents equal numbers of left and right judgements, a score of 1, 100\% leftward judgements and a score of $-1,100 \%$ rightward judgements. See Section 2 for the calculation of scores. Statistically significant effects are highlighted in 'bold' and explained in Section 2. 
Table 3

Table presenting the number of fixations and average fixation time in the left (L half) and right half (R half) of the screen when the line was judged as either shorter on the right or shorter on the left

\begin{tabular}{|c|c|c|c|c|c|c|c|c|}
\hline \multirow[t]{3}{*}{ Patient and healthy control } & \multicolumn{4}{|c|}{ Number of fixation } & \multicolumn{4}{|c|}{ Average fixation time (ms) } \\
\hline & \multicolumn{2}{|c|}{ Left shorter } & \multicolumn{2}{|c|}{ Right shorter } & \multicolumn{2}{|c|}{ Left shorter } & \multicolumn{2}{|c|}{ Right shorter } \\
\hline & $\mathrm{L}$ half & $\mathrm{R}$ half & $\mathrm{L}$ half & $\mathrm{R}$ half & $\mathrm{L}$ half & $\mathrm{R}$ half & $\mathrm{L}$ half & $\mathrm{R}$ half \\
\hline $\mathrm{LC}$ & 432 & 253 & 33 & 27 & 275 & 276 & 277 & 258 \\
\hline $\mathrm{JR}$ & 237 & 251 & 57 & 54 & 282 & 263 & 290 & 259 \\
\hline MW & 262 & 306 & 463 & 610 & 306 & 312 & 312 & 331 \\
\hline RA & 302 & 584 & 789 & 772 & 189 & 152 & 218 & 200 \\
\hline $\mathrm{MH}$ & 136 & 143 & 69 & 71 & 283 & 266 & 278 & 299 \\
\hline $\mathrm{AQ}$ & 37 & 64 & 330 & 408 & 263 & 234 & 238 & 240 \\
\hline $\mathrm{S} 1$ & 181 & 194 & 124 & 143 & 283 & 295 & 274 & 282 \\
\hline $\mathrm{S} 2$ & 317 & 326 & 298 & 316 & 228 & 196 & 225 & 191 \\
\hline S3 & 29 & 22 & 134 & 139 & 323 & 362 & 309 & 306 \\
\hline $\mathrm{S} 4$ & 8 & 12 & 77 & 130 & 251 & 202 & 250 & 218 \\
\hline S5 & 152 & 117 & 206 & 189 & 288 & 284 & 293 & 292 \\
\hline S6 & 46 & 38 & 32 & 51 & 264 & 332 & 250 & 311 \\
\hline
\end{tabular}

Data are given for patients and healthy controls for horizontal lines. Significant effects are highlighted in 'bold' and explained in Section 3.

\subsection{Size distortion}

Size distortion was assessed by calculating the percentage of times the left line was judged as shorter versus the percentage of times the right line was judged as shorter. Significant differences between these two measures were assessed with the sign test. To convey the size of the distortion effect standard contrast measures were calculated ((leftward smaller responses - rightward smaller responses)/(leftward smaller responses + rightward smaller responses)), for the same type of stimulus. This yielded three contrast scores: one for identical lines, one for lines that were $25 \%$ shorter on either the right or left and one for lines that were $12.5 \%$ shorter on either the right or left (see Table 2).

Three out of the six patients (two neglect (LC (hemianopia), JR (no hemianopia), one RCVA control patient ( $\mathrm{MH}$ (no hemianopia)) judged left lines as significantly shorter than identical right lines. This was reflected in their contrast scores. This pattern was never shown by the healthy controls. The remaining three patients (two neglect, one RCVA control) showed the opposite pattern with right lines being judged as significantly shorter than identical left lines. However, this pattern was also significant for two of the six healthy subjects (S3 and S4).

Performance on the non-identical horizontal lines gave further insight into the extent of this distortion (see Table 2): neglect patient LC with hemianopia showed the strongest effect with scores near 1 for lines that were up to $25 \%$ shorter on either the right or left. This shows a strong bias to perceive even lines that are objectively shorter on the right as shorter on the left. The contrast scores of neglect patient JR without hemianopia also reflected this with a large bias for lines that were $12.5 \%$ shorter, and his score on the lines that were $25 \%$ shorter was still outside those produced by the controls. The data on the asymmetrical lines of RCVA patient $\mathrm{MH}$ (without hemianopia), however, showed slightly weaker evidence for a leftward distortion; although his contrast score on the lines that were $12.5 \%$ shorter still reflected a bias to judge lines that were shorter on the right as shorter on the left, no bias emerged for lines that were $25 \%$ shorter on one side.

Left side underestimation of the vertical lines was not systematic, one neglect patient (RA), one RCVA control patient (MH) and one healthy subject (S5) showed the effect with a significant underestimation of the left line. Neglect patient LC showed the opposite effect with a right side underestimation but so did two of the healthy control subjects (S2 and S4). Performance on the asymmetrical lines further confirmed this pattern. All contrast scores were around 0 thus giving no evidence of left sided underestimation.

\subsection{Number of left visual field (LVF) versus right visual field (RVF) fixations}

The total number of left versus right fixations (left fixations are defined as fixations to the left of the centre of the screen and right fixations as fixations to the right of the centre of the screen) across the left and right 'shorter' judgements was analysed. A $2 \times 2 \chi^{2}$-test was used to assess the differences, using the $5 \%$ significance level throughout.

Surprisingly, none of the three patients (LC (hemianopia), JR (no hemianopia), MH (no hemianopia)) who showed a size distortion effect showed a significant relationship between number of fixations in right and left visual field and whether they judged the left or right line to be shorter (see Table 3 for fixation frequencies). Their performance was like that of the RCVA patients without a leftward size distortion effect and that of five of the six controls whose fixation patterns also did not vary systematically with their left/right judgements.

For one of the neglect patients (RA, no hemianopia) who failed to show evidence of size distortion there was 

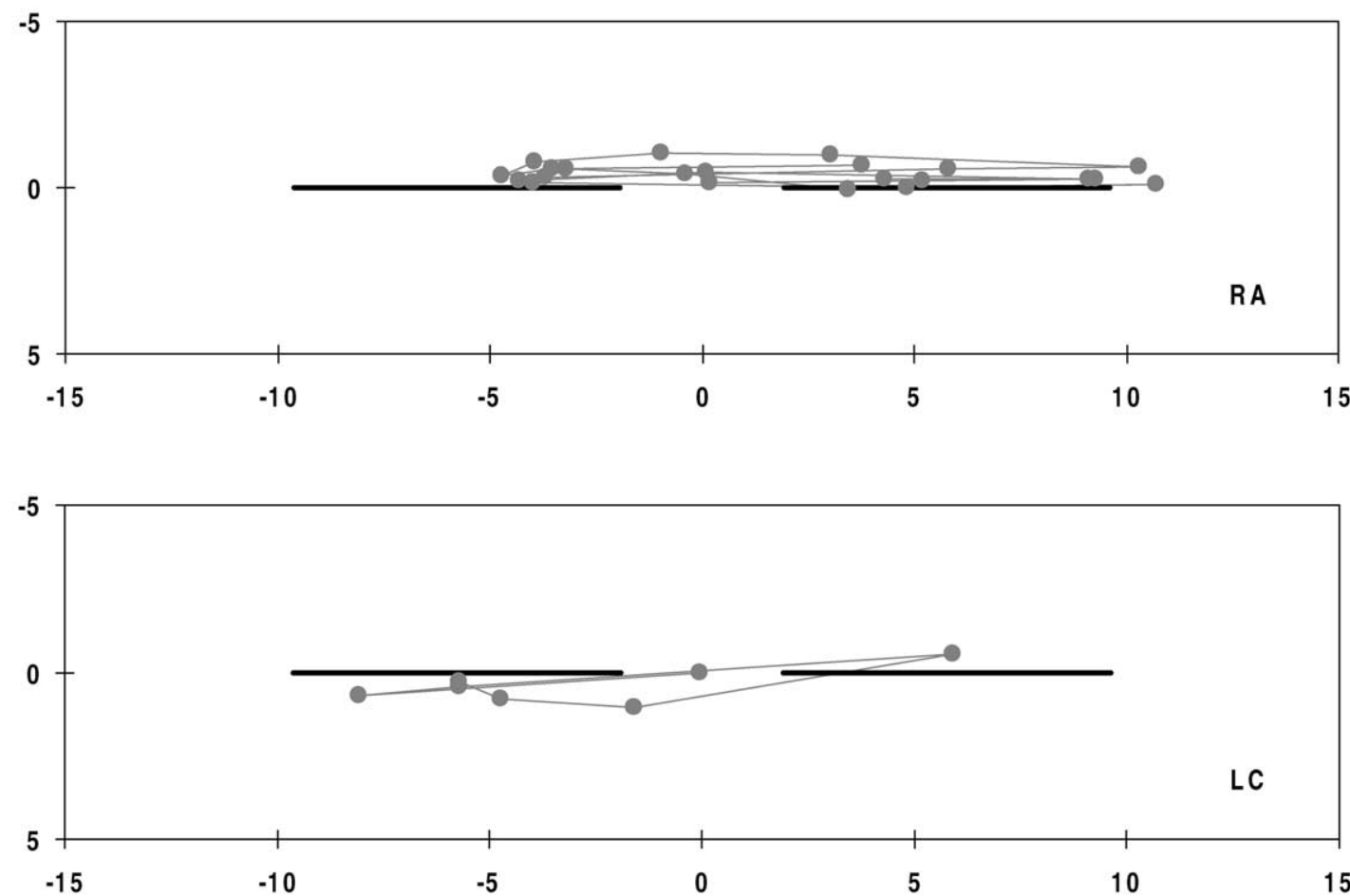

Fig. 2. Scanpaths of neglect patients LC and RA when the left line was judged to be shorter. LC has an overall strong bias to perceptually judge a leftward line as shorter whereas RA has no such bias.

a significant effect of the number of saccades in the two halves of the screen $\left(\chi^{2}(1)=61.3, P<0.01\right)$. In this case, the left/right distribution of the fixations differed between the left and right shorter judgements in that more rightward fixations were made when the identical line was judged to be shorter on the left (see Fig. 2 as an example of scanpath). A different pattern was found for control subject S6 $\left(\chi^{2}(1)=\right.$ $3.78, P=0.04)$. In his case, the left/right distribution of the fixations differed in that more rightward fixations were made for the line that was judged to be shorter on the right.

For the vertical lines, two control participants showed a greater proportion of rightward fixations when they judged the line to be shorter on the right: $\mathrm{S} 1\left(\chi^{2}(1)=5.4, P=\right.$ $0.02)$ and $\mathrm{S} 2\left(\chi^{2}(1)=24.2, P<0.01\right)$. The RCVA control patient $\mathrm{MH}$ also showed this effect $\left(\chi^{2}(1)=7.0, P<0.01\right)$ whereas the neglect patient $\mathrm{MW}\left(\chi^{2}(1)=7.1, P<0.01\right)$ showed a greater proportion of leftward fixations when the line was judged as shorter on the left.

\subsection{Average left versus right fixation time}

Average fixation time of left and rightwardly directed saccades was calculated and significant effects assessed with a general factorial ANOVA with the factors response type (left 'shorter'/right 'shorter') and fixation (left/right).

Again surprisingly, the two neglect patients (LC (hemianopia), JR (no hemianopia)) who showed the strongest underestimation of leftwardly located lines failed to show a laterality bias in terms of their average fixation time.
This lack of bias was also found in five of the six healthy control subjects, neglect patient MW (hemianopia) and the RCVA control patient AQ (no hemianopia). A significant response type by left/right fixation interaction was found for the RCVA control patient $\mathrm{MH}$ (no hemianopia) $(F(1,408)=$ $6.1, P=0.01$ ) who showed a mild size distortion effect. Paired comparisons (with a Bonferroni correction, and a resultant alpha level of 0.025) showed that he had longer duration fixations on the right side of the screen when he judged the right line to be shorter and on the left side of the screen when he judged the left side to be shorter.

A main effect of fixation left/right was found for the healthy subject $\mathrm{S} 2(F(1,1235)=21.3, P<0.01)$ across both left and right shorter responses he had longer duration fixations on the left side of the screen. Neglect patient RA showed a significant response type effect $(F(1,2442)=$ 17.9, $P<0.01$ ), he had longer fixation durations on the left when he judged the left line to the shorter. There was no such bias when he judged the right line as shorter.

For the vertical lines, four out of the six healthy subjects and three out of the six patients revealed a significant main effect of fixation left/right $(\mathrm{S} 1: F(1,588)=4.51, P=0.03$; S3: $F(1,595)=19.1, P<0.01 ;$ S4: $F(1,208)=8.4, P<$ 0.01 ; S5: $F(1,635)=10.8, P<0.01$; RA: $F(1,2411)=$ 15.9, $P<0.01$; AQ: $F(1,976)=16.9, P<0.01 ; \mathrm{MH}:$ $F(1,422)=3.9, P=0.05)$. Of the four healthy subjects, three had longer fixation durations on the left and one on the right across both response types. The RCVA control patient AQ had longer durations on the right as did the neglect 
patient RA. The RCVA control patient MH, however, fixated for longer on the left.

One neglect patient (JR) showed a main effect of response type $(F(1,875)=6.8, P<0.01)$ displaying longer fixation durations on the right part of the screen when she judged the left line to be shorter. This was not the case when the right line was judged to be shorter. Finally, S2 showed a significant response type by fixation interaction $(F(1,921)=13.7$, $P<0.01$ ), paired $t$-tests (critical alpha $=0.025$ as above) revealing that he had longer durations on the right when he judged the left line to be shorter whereas no bias was found for the right shorter judgements.

We also investigated the Horizontal Distribution of Saccades by calculating the number of fixations performed in pre-set horizontal sections of the screen, each section representing 25 pixels starting from the left end of the screen all the way through to the right end. However, for brevity this is not presented here and the reader is referred to Harvey et al. [15] for graphical presentation of this data.

\section{Discussion}

Three out of the six patients tested showed a significant size distortion effect in that they judged the left line of two identical horizontal lines as shorter than the right line, a pattern that was never present in the healthy control subjects. Two of these patients (LC, JR) also judged horizontal lines that were up to $25 \%$ shorter on the right as subjectively shorter on the left. In line with previous studies $[19,24,26]$ no systematic effects were found in the judgements of vertical lines.

We were surprised to find that the scanning patterns appeared to be unrelated to the observed size distortion effect. As revealed in both eye movement measures (number and average fixation time), the two neglect patients who showed a strong underestimation of the leftwardly placed horizontal line in comparison to the right line, gave no evidence of failing to scan the leftwardly placed line. Nevertheless, they still failed to perceive the lengths of the lines veridically, they were obviously looking without seeing size correctly. In this vein it is interesting that Dijkerman and colleagues (Dijkerman, McIntosh and Milner, personal communication) also found that the size distortion effect they observed in three neglect patients was independent of oculomotor behaviour: when asking patients to make length judgements before and after prism adaptation they found that although the deficient leftward scanning patterns were improved after prism adaptation the size misperception was not. Indeed, the scanning patterns found in these patients in particular may suggest that the observed effect could be independent of neglect as such. Although both patients did indeed have neglect symptoms their scanning patterns are not typical of neglect patients. Studies investigating scanning patterns in neglect have repeatedly found that such patients demonstrate a rightward deviation of exploratory gaze $[18,22,23]$. More specifically, although this is the first study to analyse eye movements in a length judgement task, other studies looking at scanning performance during line bisection have found rightward biases in terms of fixation distribution and fixation time in neglect patients $[2,20]$. Both MW and RA showed this scanning pattern, however, neither of them gave any evidence of a subjective size distortion.

Our single case series data gave no indication of a relationship between size misperception and hemianopia. However, this interpretation should be treated with some caution as we did not test a large number of patients and were not able to recruit patients with presence of hemianopia but absence of neglect. This was the group for which Ferber and Karnath [7] found the strongest underestimation of leftward objects. However, a recent study by Doricchi et al. [6] not only failed to replicate their finding but found the opposite effect: when testing hemianopic patients without neglect such patients showed relative overestimation of contralesional sizes and underestimation of ipsilesional sizes. They argue that Ferber and Karnath's data are problematic as all their patients were tested within 1 month (a few days in some cases) post stroke onset and were very old. It may have been that because they were so acute they were confused and unaware of their visual field defects and thus produced the unexpected behavioural patterns.

Doricchi et al. [5,6] argue that both neglect and hemianopia are prerequisites for size distortion. Hemianopia in their patients was assessed with either Humphreys or Goldmann perimetry. In our study, we simply used confrontation testing which adds to the tentativeness of any conclusions we may choose to draw from the current study. However, we found that the two patients showing the strongest effects both had hemispatial neglect but only one of them displayed a concomitant hemianopia and the patient who showed the effect mainly on the identical lines only (MH) had neither hemianopia nor neglect. It is possible that these patients had undetected field cuts but in addition, the other neglect patient with hemianopia (MW) showed no subjective size distortion. These results do not support the hypothesis that size distortion is caused by hemianopia. Moreover, in our patients, we also failed to observe the predicted hypermetric scanning in the blind field [5]. None of the three patients who showed the size distortion gave any evidence of a bias in the number of saccades they directed towards the left or right space. Only $\mathrm{MH}$ who displayed a weak effect spent more time fixating on the left when he judged the left line to be shorter. However, he had neither neglect nor hemianopia. LC who was hemianopic and had neglect, although not showing any significant biases for either number or fixation time, made more saccades to the left when judging the left line to be shorter. However, this behaviour was not significantly different from the pattern she produced when she judged the right line to be shorter. Indeed the other patient with neglect and hemianopia (MW) produced hypermetric rightward scanning independently of whether he judged the left or the right horizontal line to be shorter. 
We thus think that eye movements do not mediate the observed size distortion effect and like Milner [25,27] and Irving-Bell et al. [19] would argue that it is instead a failure to compute horizontal extent that causes the effect. Milner $[25,27]$ links this computational/representational failure to damage to parieto-temporal areas which, in the healthy brain and in the right hemisphere in particular, may integrate perceptually segregated elements of a visual scene and which receive the majority input from the visual ventral stream. In a similar vein, Irving-Bell et al. [19] propose that the ventral visual stream might be partly compromised in patients showing this size distortion effect. Bisiach et al. [4] on the other hand, although also arguing for a representational failure, link it instead to changes in neurones in areas F4 [8], area 6 [11] and especially area V6 (PO) [10] in the visual dorsal stream. In this study, interpretation in terms of lesion sites can only be speculative because of the case series methodology adopted for this investigation and we failed to find strong evidence for either explanation. The two patients who showed the effect strongly both had lesions including the right temporal lobe but two of the patients not showing misperception also had lesions including the temporal lobe. Other studies have found size misperception after parietal lesions $[4,14,28]$ but also occipital lesions $[5,7,19]$.

\section{Acknowledgements}

We would like to thank all the participants for their co-operation, Dr Lindsay Dow and Dr Peter Murphy for their help in patient access and Prof. David Perrett for most useful comments on an earlier version of this manuscript. This work was supported by a Wellcome grant to M. Harvey. B. Olk was solely funded by the Wellcome Trust (No: 050184/Z).

\section{References}

[1] Annett M. The binomial distribution of right, mixed and left handedness. Quarterly Journal of Experimental Psychology 1967;19:327-33.

[2] Barton JS, Behrmann M, Black S. Ocular search during line bisection. The effects of hemi-neglect and hemianopia. Brain 1998;121:111731.

[3] Bisiach E, Pizzamiglio L, Nico D, Antonucci G. Beyond unilateral neglect. Brain 1996;119:851-7.

[4] Bisiach E, Ricci R, Neppi-Mòdona M. Visual awareness and anisometry of space representation in unilateral neglect: a panoramic investigation by means of a line extension task. Consciousness and Cognition 1998;7:327-55.

[5] Doricchi F, Angelelli P. Misrepresentation of horizontal space in left unilateral neglect. Neurology 1999;52:1845-52.

[6] Doricchi F, Onida A, Guariglia P. Horizontal space misrepresentation in unilateral brain damage. II. Eye-centered modulation of visual misrepresentation in hemianopia without neglect. Neuropsychologia 2002;40:1118-28.

[7] Ferber S, Karnath H-O. Size perception in hemianopia and neglect. Brain 2001;124:527-36.

[8] Fogassi L, Gallese V, Fadiga L, Luppino G, Matelli M, Rizzolatti G. Coding of peripersonal space in inferior premotor cortex (area F4). Journal of Neurophysiology 1996;76:141-57.

[9] Gainotti G, Tiacci C. The relationships between disorders of visual perception and unilateral spatial neglect. Neuropsychologia 1971;9:451-8.

[10] Galletti C, Battaglini PP, Fattori P. Eye position influence on the parieto-occipital area PO (V6) of the macaque. European Journal of Neuroscience 1995;7:2486-501.

[11] Graziano MSA, Gross CG. Mapping space with neurons. Current Directions in Psychological Science 1994;3:164-7.

[12] Halligan PW, Marshall JC. Spatial compression in visual neglect: a case study. Cortex 1991;27:623-9.

[13] Harvey M, Milner AD, Roberts RC. An investigation of hemispatial neglect using the landmark task. Brain and Cognition 1995;27:5978.

[14] Harvey M, Krämer-McCaffery T, Gilchrist ID. Impaired size processing for single objects after hemispatial neglect. Neuroreport 2001;12:747-50.

[15] Harvey M, Olk B, Gilchrist, ID, Muir K. Are size distortion effects in hemispatial neglect mediated by hemianopia and/or eye-movement patterns? Cortex, in press.

[16] Heilmann KM, Valenstein E. Mechanisms underlying hemispatial neglect. Annals of Neurology 1979;5:166-70.

[17] Heilman KM, Valenstein E, Watson RT. The neglect syndrome. In: Frederiks JAM, editor. Handbook of clinical neurology, vol. 1. Clinical neuropsychology. Amsterdam: Elsevier, 1985.

[18] Hornak J. Ocular exploration in the dark by patients with visual neglect. Neuropsychologia 1992;30:547-52.

[19] Irving-Bell L, Small M, Cowey A. A distortion of perceived space in patients with right hemisphere lesions and visual neglect. Neuropsychologia 1999;37:919-25.

[20] Ishiai S, Furukawa T, Tsukagoshi H. Visuospatial processes of line bisection and the mechanisms underlying unilateral spatial neglect. Brain 1989;112:1485-502.

[21] Karnath H-O, Ferber S, Himmelbach M. Spatial awareness is a function of the temporal not the posterior parietal lobe. Nature 2001;411:950-3.

[22] Karnath H-O, Fetter M. Ocular space exploration in the dark and its relationship to subjective and objective body orientation in neglect patients with parietal lesions. Neuropsychologia 1995;33:371-7.

[23] Karnath H-O, Niemeier M, Dichgans J. Space exploration in neglect. Brain 1998;121:2357-67.

[24] Kerkhoff G. Multiple perceptual distortions and their modulation in leftsided visual neglect. Neuropsychologia 2000;38:1073-86.

[25] Milner AD. Streams and consciousness: visual awareness and the brain. Trends in Cognitive Sciences 1998;2:25-30.

[26] Milner AD, Harvey M. Distortion of size perception in visuospatial neglect. Current Biology 1995;4:85-9.

[27] Milner AD, Harvey M, Pritchard CL. Visual size processing in spatial neglect. Experimental Brain Research 1998;123:192-200.

[28] Olk B, Harvey M. Effects of visible and invisible cueing on line bisection and landmark performance in hemispatial neglect. Neuropsychologia 2002;40:282-90.

[29] Vallar G. Spatial neglect in humans. Trends in Cognitive Sciences 1998;2:87-96.

[30] Wilson BA, Cockburn J, Halligan P. Behavioural inattention test. Titchfield, Hampshire: Thames Valley Test Company, 1987. 